\title{
Dysphagia Lusoria due to diffuse idiophatic skeletal hyperostosis (DISH): Case Report
}

Rafaelle Galiotto Furlan', Ricardo Maraschim², Jonathan Soldera ${ }^{3,4}$, Raul Ângelo Balbinot ${ }^{3,5 *}$, Silvana Sartori Balbinot ${ }^{3,5}$, Rafael Sartori Balbinot $^{6}$ and Alana Zulian Terres ${ }^{1}$

${ }^{1}$ Clinical Gastroenterology, Faculty of Medicine, Universidade de Caxias do Sul, Brazil

${ }^{2}$ Internal Medicine, Faculty of Medicine, Universidade de Caxias do Sul, Brazil

${ }^{3}$ Clinical Gastroenterology, Faculty of Medicine, Universidade de Caxias do Sul, Brazil

${ }^{4}$ Universidade Federal de Ciências da Saúde de Porto Alegre, Brazil

${ }^{5}$ Universidade de São Paulo, Brazil

${ }^{6}$ Faculty of Medicine, Universidade de Caxias do Sul, Brazil

\begin{abstract}
Diffuse idiopathic skeletal hyperosteosis (DISH) (or Forestier's Disease) is an ossifying diathesis characterized by spinel and peripheral enthesopathy. This disease is more prevalent in men, usually after the 5 th decade of life. In this article, it is reported the case of a 78-year-old man presenting with progressive dysphagia, weight loss, malnutrition and dysphonia. Computed tomography showed an important bony prominence compressing the first portion of the esophagus, in the level of C3C4. The preferred treatment is the excision of the osteophyte.
\end{abstract}

\section{Introduction}

Diffuse idiopathic skeletal hyperosteosis (DISH) (or Forestier's Disease) is an ossifying diathesis characterized by spinel and peripheral enthesopathy. It generally compromises the axial skeleton and in some cases the peripheral joints. This disease is more prevalent in men than women and the incidence increases with age - it is rarely seen before the $4^{\text {th }}$ decade of life. It becomes symptomatic when the hyperosteosis involves the anterior margin of the cervical vertebrae, causing dysphonia, dyspnea and/or dysphagia. First described in 1950 by Forestier and Rotes-Querol [1] and reviewed in 1970 by Resnick et al. [2], the literature does not set a standard for the diagnosis or treatment of DISH. The purpose of this paper is to report a case of dysphagia lusoria due to DISH.

\section{Case report}

A 78-year-old male patient was admitted in the emergency department with a complaint of progressive dysphagia for solids and then for liquids with two years of evolution and weight loss of about 40 pounds. He described a worsening of his condition in the previous four months, a period in which gagging and aspiration of food became frequent. He also complained of cervical and upper limbs morning stiffness with diffuse pains in the spine which began around 30 years ago. He did not have any previous diagnosis or was using any medication.

He was previously investigated before admission with a chest radiography, which came back normal. A barium swallow (Figures 1 and 2) was performed, which showed narrowing of the upper esophagus and gastro-esophageal reflux of contrast. An upper digestive endoscopy (UDE) found a tortuous esophagus presenting tertiary waves. An esophageal manometry was not able to be performed due to difficulty of catheter progression.
Due to the appearance of dysphonia and aggravation of dysphagia, he was admitted. Patient presented malnourished and dehydrated, with muscle flaccidity, cervical and axial skeletal rigidity. Another UDE was performed (Figure 3), which showed a bulge in the hypopharynx due to external compression - dysphagia lusoria. Computed Tomography (CT) (Figures 4 and 5) presented degenerative alterations affecting the cervical vertebrae with osteophytosys, reduction of discal spaces, subchondral cysts and anterior bridges syndesmotic. At the C3-C4 level, there was a large anterior osteophyte, which compressed the upper esophagus at the level of the vestibular fold (Figures 6,7 and 8). Due to malnutrition and repetitive aspiration pneumonias, feeding was started through a nasoenteral tube. Patient was referred for orthopedic surgeon to perform definitive treatment with resection of the cervical osteophyte.

\section{Discussion}

The prevalence of DISH is estimated at 10 to $35 \%$ of male patients older than 70 years, with propensity for Caucasians [3]. As reported by Verlaan et al, the mean age at the diagnosis was 68.9 years and the male-to-female ratio was 6:1 [4].

The pathogenesis of DISH remains unknown, but involves mechanical factors, dietary contributions, drugs, environmental exposures, metabolic conditions (obesity, arterial hypertension, diabetes mellitus and metabolic syndrome) and genetic (few

Correspondence to: Raul Ângelo Balbinot, Clinical Gastroenterology, Faculty of Medicine, Universidade de Caxias do Sul, Universidade de São Paulo, Brazil; E-mail: raulbalbinotti@terra.com.br

Received: September 29, 2017; Accepted: October 30, 2017; Published: November 02, 2017 


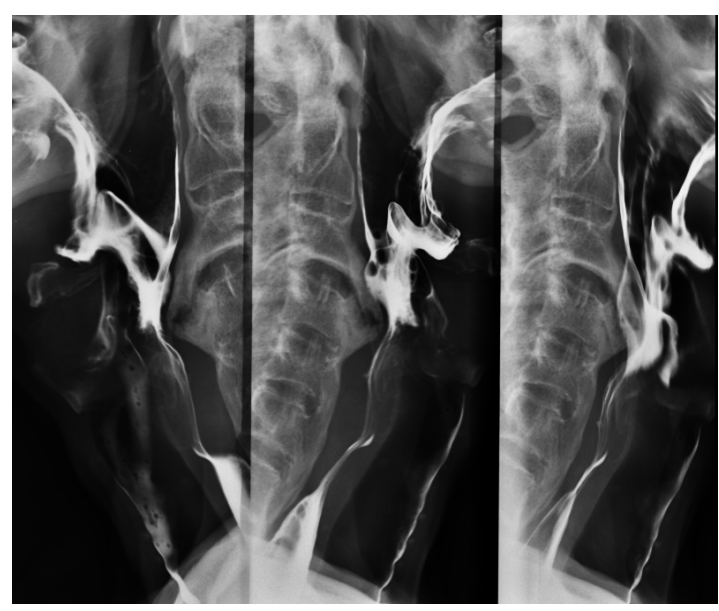

Figure 1. Barium Swallow: Esophageal Narrowing

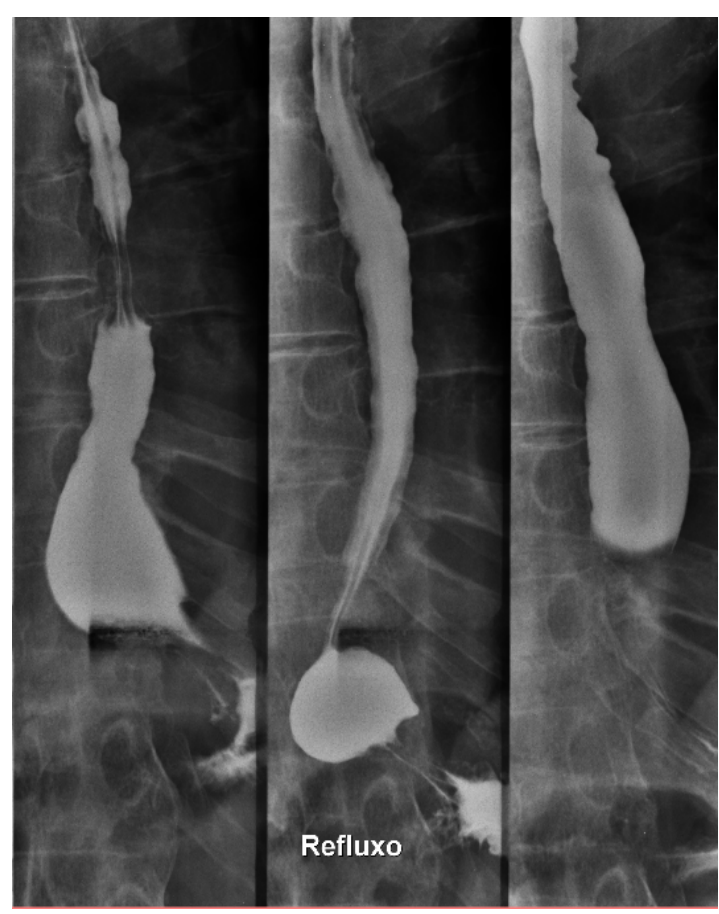

Figure 2. Barium Swallow: Gastroesophageal Reflux

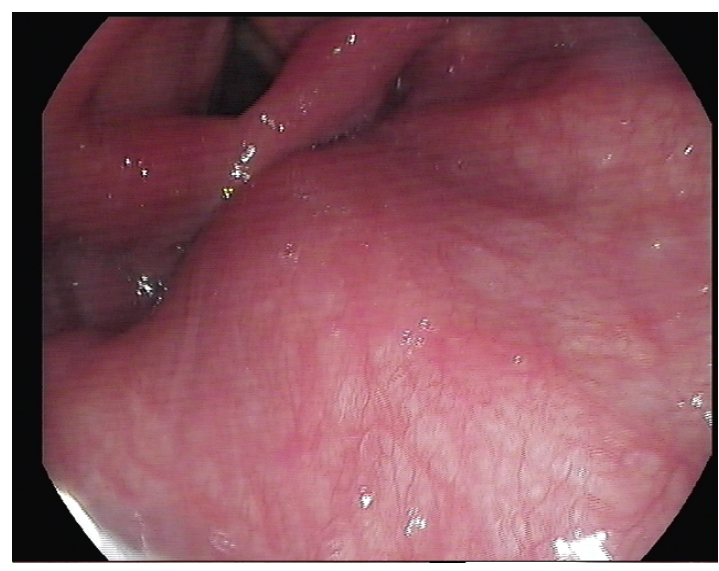

Figure 3. Upper Digestive Endoscopy: Bulge in the hypopharynx

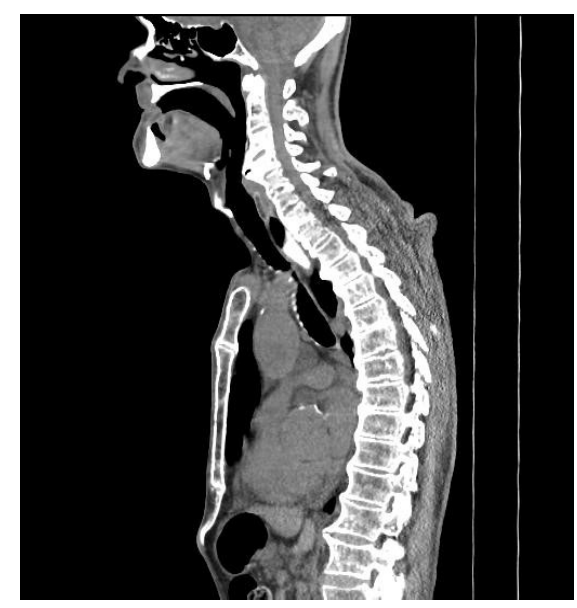

Figure 4. Computed Tomography: diffuse skeletal hyperostosis

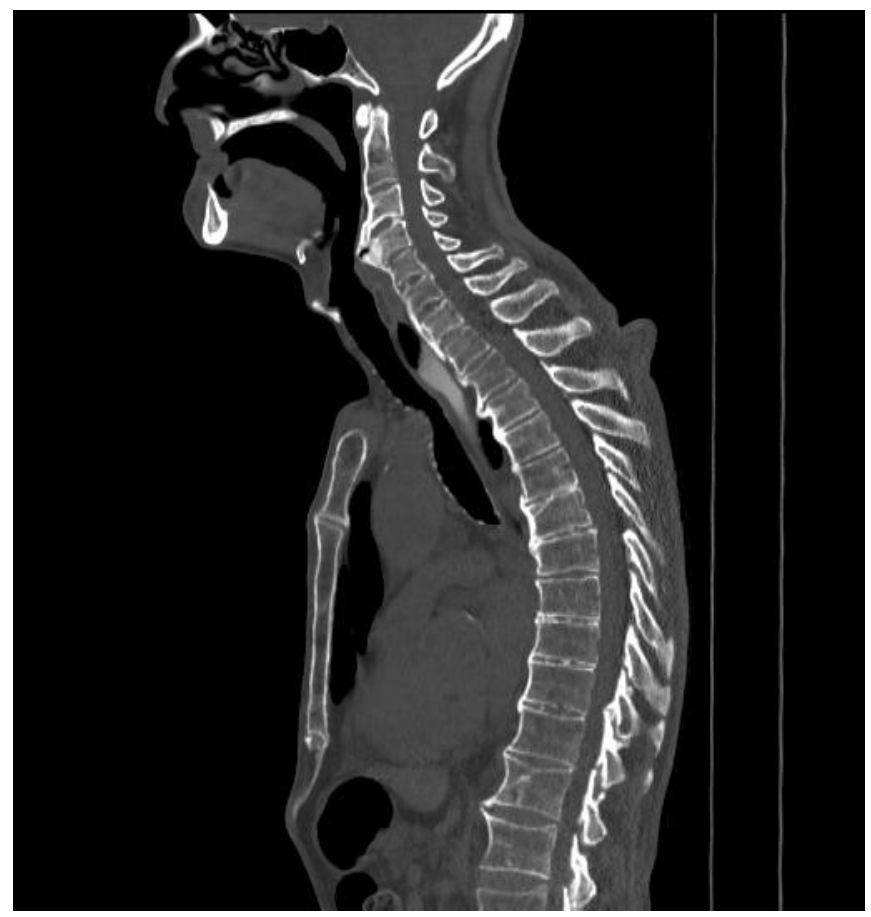

Figure 5. Computed Tomography: diffuse skeletal hyperostosis

descriptions of familial cases) [5]. The main hypothesis is based on the excess of growth factors that might induce transformation of mesenchymal cells into fibroblasts and osteoblasts such as insulin, insulin-like growth factor1, prostaglandin I2 and endothelin-1 [6-7]. However, such associations have not been definitively established and are a potential field for further investigation.

Although DISH is asymptomatic in most individuals, this condition might indicate underlying metabolic disease. Spinal or extraspinal ossifications can sometimes lead to symptoms including pain, stiffness, a reduced range of articular mobility, and dysphagia lusoria, and increases the risk of unstable spinal fractures [8]. The most frequent level of involvement related to dysphagia is C5-C6 followed by $\mathrm{C} 4-\mathrm{C} 5$ and $\mathrm{C} 2-\mathrm{C} 3$ is the less common level affected [9]. The anterior compression can also lead to odynophasia, dysphonia, feeling of foreign body in the throat and, rarely, some more severe complications as airway aspiration of liquids or solids and obstructive sleep apnea [10]. 


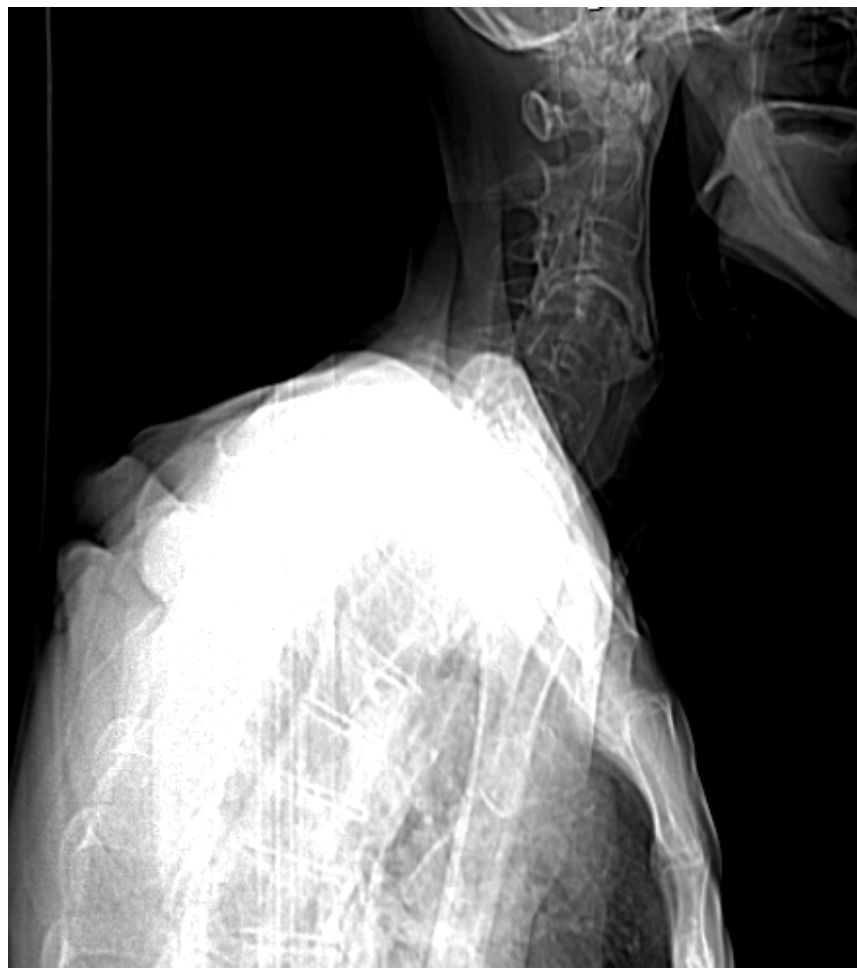

Figure 6. Computed Tomography: anterior cervical osteophyte formation

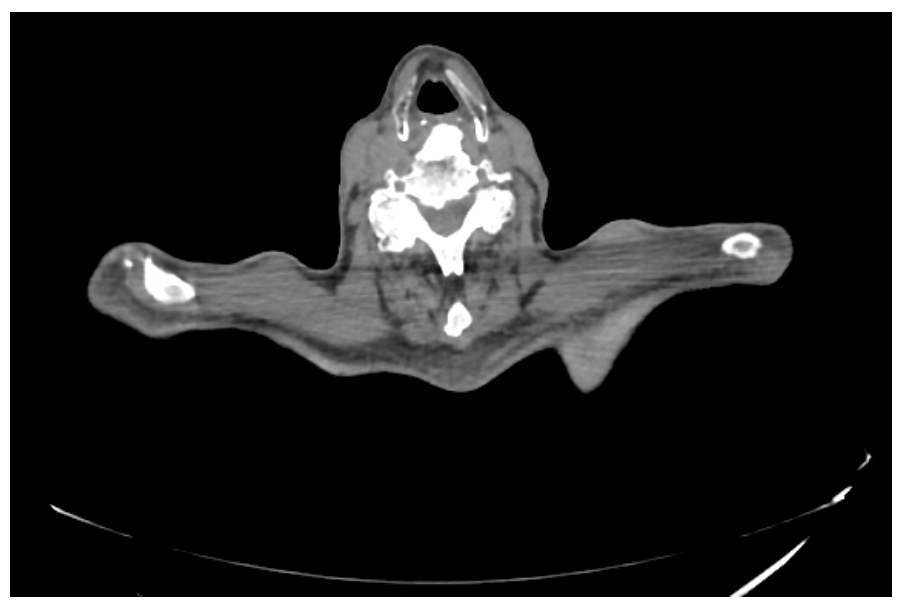

Figure 7. Computed Tomography: anterior cervical osteophyte formation.

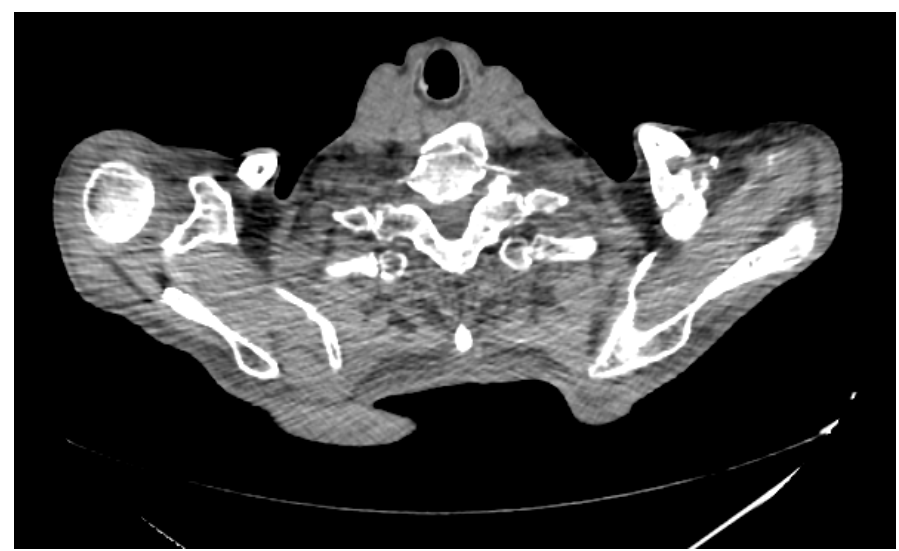

Figure 8. Computed Tomography: anterior cervical osteophyte formation.
The published criteria for the diagnosis of DISH have been proposed by Resnick and Niwayama in 1976 (Table 1) [8]. They are based on the presence of flowing calcification and ossification along the anterolateral facade of at least four contiguous vertebral bodies with or without associated localized pointed excrescences at the intervening vertebral body-intervertebral disc junctions; the presence of relative preservation of intervertebral disc height in the involved vertebral segment and the absence of extensive radiographic changes of "degenerative" disc disease, including vacuum phenomena and vertebral body marginal sclerosis; the absence of apophyseal joint bony ankyloses and sacroiliac joint erosion, sclerosis, or intraarticular osseous fusion. Utsinger has recommended different criteria in 1985 (Table 2) [2] which are based on continuous ossification along the anterolateral facade of at least four contiguous vertebral bodies, primarily in the thoracolumbar spine. Ossification begins as a fine, ribbon-like wave of bone but commonly develops into a broad, bumpy, buttress-like band of bone (definitive criteria); continuous ossification along the anterolateral aspect of at least two contiguous vertebral bodies and symmetrical and peripheral enthesopathy involving the posterior heel, superior patella, or olecranon, with the entheseal new bone having a well-defined cortical margin (probable criteria) $[2,8]$.

The definitive imaging characteristics of DISH are flowing osteophytes mainly in the thoracic spine. However, a number of conditions may produce bony excrescences similar to those observed with DISH, as ankylosing spondylitis (AS). The formation of thick bony spinal bridges from along the anterior longitudinal ligament in a more horizontal orientation and mainly on the right side is commonly encountered with DISH, and the delicate vertically oriented syndesmophytes are the hallmark of AS [11,12].

There have been no controlled studies examining the effectiveness of any therapy for this disorder. Therefore, the treatment of DISH is symptomatic and empiric. The approach of pain involves the use of analgesics as local or systemic non-steroidal anti-inflammatory drugs, physiotherapeutic modalities and lifestyle changes, such as diet programs. Due to the co-morbidities that often accompany DISH, it

Table 1. Resnick and Niwayama (1976)

1. The presence of flowing calcification and ossification along the anterolateral aspect of at least four contiguous vertebral bodies with or without associated localized pointed excrescences at the intervening vertebral body-intervertebral disc junctions.

2. The presence of relative preservation of intervertebral disc height in the involved vertebral segment and the absence of extensive radiographic changes of "degenerative" disc disease, including vacuum phenomena and vertebral body marginal sclerosis.

3. The absence of apophyseal joint bony ankylosis and sacroiliac joint erosion, sclerosis, or intraarticular osseous fusion.

\section{Table 2. Utsinger (1985)}

1. Continuous ossification along the anterolateral aspect of at least four contiguous vertebral bodies, primarily in the thoracolumbar spine. Ossification begins as a fine, ribbon-like wave of bone but commonly develops into a broad, bumpy, buttress-like band of bone

2. Continuous ossification along the anterolateral aspect of at least two contiguous vertebral bodies.

3. Symmetrical and peripheral enthesopathy involving the posterior heel, superior patella, or olecranon, with the entheseal new bone having a well-defined cortical margin

Exclusions:

i) abnormal disc space height in the involved áreas

ii) apophyseal joint ankylosis

Categories of DISH according to the Utsinger criteria are:

Definite $=$ criterion 1

Probable $=$ criteria 2 and 3 
has been suggested the avoidance of medications that might enhance insulin secretion such as sulfonylureas, $\beta$-adrenergic blockers or thiazide diuretics [13]. Surgery is occasionally required to improve dysphasia cause by large cervical spurs [14].

\section{Conclusion}

DISH (or Forestier's disease) is a systemic condition that affects people over 50 years. This condition often indicates underlying metabolic disease. The presence of spinal or extraspinal ossifications can sometimes lead to symptoms including pain, stiffness, a reduced range of articular motion, and dysphagia lusoria - an uncommon symptom that may result from external compression of the esophagus by large anterior cervical osteophyte. Although symptomatic treatment is recommended, in cases of persistent symptoms osteophytectomy is indicated.

\section{References}

1. Forestier J, Rotes-querol J (1950) Senile ankylosing hyperostosis of the spine. Ann Rheum Dis 9: 321-330. [Crossref]

2. Resnick D, Niwayama G (1976) Radiographic and pathologic features of spinal involvement in diffuse idiopathic skeletal hyperostosis (DISH). Radiology 119: 559. [Crossref]

3. Pulcherio JO, Velasco CM, Machado RS, Souza WN, Menezes DR (2014) Forestier's disease and its implications in otolaryngology: Literature review. Braz J Otorhinolaryngol 80:161. [Crossref]
4. Verlaan JJ, Boswijk PF, de Ru JA, Dhert WJ, Oner FC (2011) Diffuse idiopathic skeletal hyperostosis of the cervical spine: An underestimated cause of dysphagia and airway obstruction. Spine J 11:1058-1067. [Crossref]

5. Forestier J, Lagier R (1971) Ankylosing hyperostosis of the spine. Clin Orthop Relat Res 74: 65-83. [Crossref]

6. Denko CW, Malemud CJ (2006) Body mass index and blood glucose: correlations with serum insulin, growth hormone, and insulin-like growth factor-1 levels in patients with diffuse idiopathic skeletal hyperostosis (DISH). Rheumatology International 26:292-297.

7. Denko CW, Boja B, Malemud CJ (2003) Intra-erythrocyte deposition of growth hormone in rheumatic diseases. Rheumatol Int 23: 11-14. [Crossref]

8. Utsinger PD (1985) Diffuse idiopathic skeletal hyperostosis. Clin Rheum Dis 11: 325351. [Crossref]

9. Hilding DA, Tachdjian MO (1960) Dysphagia and hypertrophic spurring of the cervical spine. N Engl J Med 263: 11-14.

10. Kmucha ST, Cravens RB Jr (1994) DISH syndrome and its role in dysphagia. Otolaryngol Head Neck Surg 110: 431-436. [Crossref]

11. Olivieri I, D’Angelo S, Cutro MS, Padula A, Peruz G, et al. (2007) Diffuse idiopathic skeletal hyperostosis may give the typical postural abnormalities of advanced ankylosing spondylitis. Rheumatology 46: 1709-1711. [Crossref]

12. Olivieri I, D’Angelo S, Palazzi C, Padula A (2013) Spondyloarthritis and diffuse idiopathic skeletal hyperostosis: two different diseases that continue to intersect. $J$ Rheumatol 40: 1251-1253. [Crossref]

13. Mader R (2005) Current therapeutic options in the management of diffuse idiopathic skeletal hyperostosis. Expert Opin Pharmacother 6: 1313-1318. [Crossref]

14. Castellano DM, Sinacori JT, Karakla DW (2006) Stridor and dysphagia in diffuse idiopathic skeletal hyperostosis (DISH). Laryngoscope 116: 341-344. [Crossref]

Copyright: (C2017 Furlan RG. This is an open-access article distributed under the terms of the Creative Commons Attribution License, which permits unrestricted use, distribution, and reproduction in any medium, provided the original author and source are credited. 